1 FLAgellum Member 8 modulates extravascular trypanosome distribution in the

2 mammalian host

3

4 Estefanía Calvo Alvarez ${ }^{1 *}$, Christelle Travaillé ${ }^{1}$, Aline Crouzols ${ }^{1}$ and Brice Rotureau*1,2

5

$6 \quad{ }^{1}$ Trypanosome Transmission Group, Trypanosome Cell Biology Unit, Department of

7 Parasites and Insect Vectors and INSERM U1201, Institut Pasteur, Paris, France

82 Trypanosome Transmission Group, Parasitology Lab, Institut Pasteur of Guinea,

9 Conakry, Guinea.

10

$11{ }^{*}$ Corresponding author: estefaniacalvoalvarez@gmail.com, brice.rotureau@pasteur.fr 12

13 Keywords: Trypanosoma brucei, FLAM8, flagellum, extravascular, dissemination,

14 skin

15

16 Running tittle: FLAM8 modulates extravascular trypanosome dissemination

17

184 Figures, 5 Supplementary Figures and 1 Table 


\section{Take away:}

- FLAM8 is dispensable in vitro for survival, growth, motility and differentiation of $T$.

21 brucei.

22 - FLAM8 depletion does not affect parasitemia and bloodstream form differentiation 23 in vivo.

24 - FLAM8 modulates the extravascular dissemination of trypanosomes in the 25 mammalian host, especially in the skin. 


\section{Summary}

27 The African trypanosome flagellum is an essential organelle in multiple aspects of the parasites' development. Here, we investigated the role of a flagellar protein termed FLAgellar Member 8 (FLAM8) that is specifically distributed along the entire flagellum in trypanosomes stages of the vertebrate host. Analyses of knockdown and knockout trypanosomes demonstrated that FLAM8 is not essential in vitro for survival, growth, motility and slender to stumpy differentiation. Functional investigations in experimental infections showed that FLAM8-deprived trypanosomes are able to establish and maintain the infection in the blood circulation, and to differentiate into insect transmissible forms. However, quantitative bioluminescence imaging revealed that FLAM8-null parasites exhibit an impaired dissemination in the extravascular compartment, that is partially restored by the addition of a single rescue copy of FLAM8. Interestingly, among all dissected organs scrutinized individually, only the skin of mice infected with FLAM8-deprived parasites showed a significant reduction in extravascular trypanosome population as compared to mice infected with parental controls. To our knowledge, FLAM8 is the first example of a flagellar protein that modulates $T$. brucei parasite distribution in the host tissues, contributing to the maintenance of extravascular parasite populations in mammalian anatomical niches, especially in the skin. 
Introduction

Trypanosoma brucei is an extracellular parasite responsible for African trypanosomiases in sub-Saharan Africa, a group of neglected tropical diseases including sleeping sickness in humans and nagana in cattle. African trypanosomes are blood and tissue-dwelling protists transmitted by the bite of the blood-feeding tsetse fly (Glossina genus). In the mammalian host, parasites have to face different microenvironments, including deadly challenges by multiple types of host immune responses and the variable availability of carbon sources. This requires major morphological and metabolic adaptations, driven by the activation of specific gene expression programs, that are critical for life-cycle progression (MacGregor, Szoor, Savill, \& Matthews, 2012; Ooi \& Bastin, 2013; Smith, Bringaud, Nolan, \& Figueiredo, 2017). Recently, the importance of extravascular tropism for T. brucei has been rediscovered and emphasized in animal models: in addition to the brain, parasites actually occupy most mammalian tissues, especially the skin and the adipose tissues (Caljon et al., 2016; Capewell et al., 2016; Trindade et al., 2016). However, when,

60 where and how trypanosomes adapt to survive within these extravascular environments is not understood yet.

The trypanosome flagellum is an essential organelle anchored along the surface of the cell body and present in all stages of its development (Rotureau, Subota, \& Bastin, 2011). It is essential for parasite viability (Broadhead et al., 2006), cell division and morphogenesis (Kohl, Robinson, \& Bastin, 2003), attachment to the tsetse salivary glands (Tetley \& Vickerman, 1985) and motility (Langousis \& Hill, 2014). In the insect host, the flagellum remains at the forefront of the cell and is likely to be involved in sensory and signaling functions required for host-parasite interactions (Roditi, 
Schumann, \& Naguleswaran, 2016; Rotureau, Morales, Bastin, \& Spath, 2009). In the mammalian host, flagellar motility was shown to be critical for establishment and maintenance of bloodstream infection (Shimogawa et al., 2018). Nevertheless, the overall contributions of the trypanosome flagellum to parasite tropism and spatiotemporal dissemination dynamics in the mammalian host are important aspects that still remained to be explored more in depth.

Our proteomic analysis of intact flagella purified from the insect stage of the parasite identified a group of flagellar membrane and matrix proteins with unique patterns and dynamics (Subota et al., 2014). Amongst them, one large protein (3,075 amino acids) termed FLAgellar Member 8 (FLAM8) is present only at the distal tip of the flagellum in the insect procyclic form (Bertiaux, Morga, Blisnick, Rotureau, \& Bastin, 2018; Fort, Bonnefoy, Kohl, \& Bastin, 2016; Subota et al., 2014). Interestingly, it is redistributed along the entire length of the flagellum in mammalian bloodstream forms (BSF), including in stumpy transmissible stages (Calvo-Alvarez et al., in revision), which may imply a specific function for FLAM8 in the vertebrate host. Therefore, we hypothesized that the stage-specific redistribution of FLAM8 could be involved in host-parasite interactions, possibly affecting parasite homing and dynamics in host tissues. Here, we investigated the possible roles of FLAM8 in vitro in terms of survival, proliferation, motility and differentiation. In addition, experimental infections in mice monitored by bioluminescence imaging allowed us to decipher the involvement of FLAM8 in parasite dissemination and spreading in both the intra- and extravascular compartments of the mammalian host. Potential consequences for parasite transmission along with cellular mechanisms of tissue tropism are further discussed. 


\section{Results}

FLAM8 RNAi silencing does not affect parasite survival in vitro and in vivo.

The differential distribution of FLAM8 in the flagellum of the different trypanosome stages (Calvo-Alvarez et al., in revision) raises the question of its specific functions during the parasite cycle. In order to investigate the potential role(s) of FLAM8 in the mammalian host, BSF were first engineered for inducible RNAi knockdown of FLAM8 in a monomorphic strain expressing a mNeonGreen-tagged version of FLAM8. In order to monitor their behavior in mouse by whole-body imaging approaches, these FLAM8::mNG FLAM8 ${ }^{R N A i}$ mutants were subsequently transformed to overexpress a chimeric triple reporter protein (Calvo-Alvarez, Cren-Travaillé, Crouzols, \& Rotureau, 2018). Upon RNAi induction with tetracycline for $72 \mathrm{~h}$, FLAM8 expression was reduced by $60 \%$ at the mRNA level (Fig. 1A) and became undetectable at the protein level by immunofluorescence (Fig. 1B). Parasite growth was monitored over 6 days upon induction of RNAi and no impact on proliferation was observed (Fig. 1C), which indicates that FLAM8 is not essential for survival of BSF parasites in cell culture conditions.

Then, the linear correlation between the emitted bioluminescence and the number of parasites was assessed in an IVIS Spectrum imager prior to in vivo challenge (Fig. S1). In order to get insights into the function of FLAM8 in the mammalian host, groups of male BALB/c mice were infected by the intraperitoneal route with $10^{5} \mathrm{BSF}$ parasites of the parental, non-induced and induced cell lines (Fig. 2). In vivo RNAi silencing of FLAM8 was maintained in mice by the addition of doxycycline in sugared drinking water $48 \mathrm{~h}$ prior infection and until the end of the experiment. The course of the infection was monitored daily by i) quantifying the parasitemia, and ii) acquiring the bioluminescent signal emitted by the parasites in the entire organism with an IVIS 
117 Spectrum imager. The number of parasites in the extravascular compartment at a

118 given timepoint can be extrapolated by subtracting the known number of trypanosomes

119 in the vascular system (parasitemia $x$ blood volume, according to body weight) from

120 the total number of parasites in the organism (total bioluminescence). No differences

121 were detected neither in the establishment of the infection and the subsequent

122 variations in the number of intravascular parasites (Fig. 2A), nor in the number of 123 parasites occupying extravascular tissues (Fig. 2B) and the animal survival (not

124 shown). Similar population profiles were observed over the course of the infection for

125 both IV and EV parasites in each of the three independent groups of infected mice

126 (Fig. 2, C-E). Finally, in the extravascular compartment, no significant differences were

127 detected in terms of parasite dissemination over the entire animal body in any of the

128 groups tested (Fig. 2, F-G).

129 FLAM8 knockout affects trypanosome spreading in the mammalian host.

130 Considering that i) FLAM8 RNAi silencing efficiency was only partial (40\% FLAM8

131 mRNA left after $72 \mathrm{~h}$ of induction), ii) the efficacy of doxycycline-induced FLAM8

132 repression could have been even lower in vivo, and that iii) the parental strain used for

133 this first strategy was monomorphic (i.e. unable to differentiate into tsetse adapted

134 stumpy stages), we reasoned that a gene knockout approach in a pleomorphic strain

135 would be more appropriate to evaluate the potential role(s) of FLAM8 during the

136 mammalian host infection. Therefore, a $\triangle F L A M 8$ knockout cell line was generated in

137 pleomorphic BSF trypanosomes by homologous recombination. The replacement of

138 both FLAM8 alleles by distinct resistance cassettes was verified by whole-genome

139 sequencing and PCRs (Fig. S2, A-B). The trypanosome cell lines generated were

140 further transformed to express the chimeric triple reporter construct as previously 141 described (Calvo-Alvarez et al., 2018), and the linear correlation between the 
142 bioluminescence signal and the total number of parasites was analyzed for all strains

143 (Fig. S3). In $\triangle F L A M 8$ knockout parasites where a rescue copy of one FLAM8 allele

144 was added back into its endogenous locus, the FLAM8 distribution was restored along

145 the entire flagellum, as assessed by immunofluorescence analysis (Fig. 3A). No impact

146 on parasite growth in culture conditions was observed (Fig. 3B). Next, we investigated

147 whether the loss of FLAM8 could have impacted the total length of the flagellum based

148 on the measurement of the signal obtained with the axonemal marker mAb25: no

149 difference was observed among BSF lines (Fig. 3C). In addition, the absence of

150 FLAM8 did not affect parasite motility in vitro, neither in terms of speed nor in linearity

151 (Fig. 3D-E, respectively). Compatible with our previous observations after RNAi

152 silencing, these results show that pleomorphic trypanosomes tolerated the loss of

153 FLAM8 in vitro.

154 Then, functional investigations in the mammalian host were performed by infecting

$155 \mathrm{BALB} / \mathrm{c}$ mice either with the pleomorphic parental strain, three distinct $\triangle F L A M 8$

156 knockout subclones or one $\triangle F L A M 8$ strain bearing a rescue copy of FLAM8 (Fig. 4).

157 The infections were monitored daily during 4 weeks by quantifying the parasitemia and

158 the bioluminescence signals emitted from whole animals (Fig. 4A). Null mutant 159 parasites were able to establish an infection in the bloodstream as well as in the

160 extravascular compartment. In the blood circulation, the absence of FLAM8 did not

161 prevent the in vivo differentiation of proliferative slender into transmissible stumpy

162 parasites in the intravascular compartment (Fig. 4B), compatible with observations

163 upon in vitro differentiation (Fig. S4, A-B). In addition, freshly-differentiated FLAM8-

164 deprived stumpy parasites were able to further differentiate and maintain in vitro as

165 procyclic trypanosomes (Fig. S4C). Within the vasculature, the overall amounts of

166 parasites throughout the whole experimental infection were similar in all strains (Fig. 
$4 C)$, yet significant differences between the three $\triangle F L A M 8$ subclones as compared to parental-infected BALB/c mice were observed at days 5, 6 and 7, coinciding with the first peak of parasitemia (Fig. 4C). On the other hand, quantitative analyses of

170 extravascular parasites showed a different scenario. Unlike in the intravascular 171 compartment, significantly lower numbers of extravascular trypanosomes were observed between day 5 to 12, and from day 19 post-infection until the end of the experiment at day 27 , evidencing an impaired extravascular colonization for $\triangle F L A M 8$ parasites as compared to parental controls (Fig. 4D). To note, distribution profiles of

175 all pleomorphic strains were different from those observed in mice infected with monomorphic parasites: larger amounts of trypanosomes were seen occupying the extravascular compartment reaching up to $5-8 \times 10^{9}$ parasites, while the maximum

178 amount found in the bloodstream never exceeded $7 \times 10^{7}$ trypanosomes (Fig. S5).

179 Furthermore, the subsequent quantification of the parasite spreading over the whole

180 animal bodies showed that the depletion of FLAM8 resulted in a partially yet 181 significantly impaired dissemination of $\triangle F L A M 8$ null mutant parasites in the 182 extravascular compartment (days 5 to 13, 19 and 27 post-infection). This was mostly 183 restored in trypanosomes bearing a rescue copy of FLAM8 (Fig. 4E). Next, individual 184 imaging of dissected organs was performed at the end of the experimental infection in 185 order to estimate the amount of extravascular parasites in each organ. This was 186 obtained by normalizing the total number of parasites within each organ (calculated 187 from the bioluminescence) to the parasitemia level in the corresponding mouse at this 188 last time point (Fig. 4F). At day 27, only the extravascular parasite colonization was 189 significantly different between mice groups. Interestingly, the reduced distribution of 190 FLAM8-deprived trypanosomes in the extravascular compartment was not detectable 191 in most organs. Only the skin of $\triangle F L A M 8$-infected animals presented a statistically 
192 significant difference as compared to mice infected with the parental strain (Fig. 4F).

193 In conclusion, these results indicate that FLAM8 is involved in the modulation of

194 trypanosome distribution in the extravascular compartment of the mammalian host,

195 especially in the skin. 


\section{Discussion}

197 The differential localization of FLAM8 from the very distal tip in tsetse midgut procyclic

198 parasites to the entire flagellum length in the mammalian-infectious stages (Calvo-

199 Alvarez et al., in revision) prompted us to speculate that FLAM8 could play a specific 200 role in the mammalian host. Here, we present for the first time a connection of a

201 flagellar protein with the efficiency of trypanosomes to distribute outside the host

202 vasculature, especially in the skin. Quantitative analyses of experimental animal

203 infections monitored by bioluminescence imaging showed that the absence of FLAM8

204 impaired parasite dissemination in the host extravascular compartment over the time 205 of the infection, which was mostly recovered by the integration of a single rescue copy 206 of the FLAM8 gene in the endogenous locus.

\section{1. FLAM8 and trypanosome transmission}

208 Extravascular trypanosomes occupy the interstitial space of several organs, including 209 the central nervous system, testes, adipose tissues and skin (Biteau et al., 2016;

210 Caljon et al., 2016; Capewell et al., 2016; Carvalho et al., 2018; Goodwin, 1970;

211 Trindade et al., 2016). The relevance of skin-dwelling trypanosomes in parasite

212 transmission was demonstrated by xenodiagnosis experiments, early after the 213 infective bite (Caljon et al., 2006), or later in the infection (Capewell et al., 2016), even

214 in the absence of detectable parasitemia. More recently, the presence of extravascular

215 trypanosomes was confirmed in the skin of confirmed and suspected cases of sleeping 216 sickness (Camara et al., 2020). Here, the impaired spreading of FLAM8-null parasites 217 over the extravascular compartment, especially in the skin, would mathematically 218 reduce the probability for parasites to be ingested by tsetse flies.

219 In the bloodstream, the balance between proliferative slender parasites and tsetse220 adapted stumpy forms responds to a quorum sensing mechanism involving the 
221 production of oligopeptides and their reception through a specific transporter (Rojas et al., 2018). Although stumpy proportions were not evaluated over the entire course of

223 the infection, the absence of FLAM8 did not alter the ability of the parasites to

224 differentiate into transmissible stumpy forms at the peak of parasitemia, showing that

225 the protein is most probably not involved in this process. In addition, the ability of

226 FLAM8-deprived stumpy BSF to differentiate into procyclics in vitro suggests that

227 stumpy cells present in the blood and in the dermis could further develop upon 228 ingestion by a tsetse fly.

229 2. On the possible cellular function(s) of FLAM8

230 Proliferative slender trypanosomes are highly mobile (Bargul et al., 2016) and this 231 motility was proved to influence virulence in vivo. For instance, the lack of propulsive 232 motility in flagellar dynein LC1 knockout mutants resulted in the inability of 233 trypanosomes to establish an infection in the bloodstream (Shimogawa et al., 2018).

234 Here, FLAM8 silencing in pleomorphic BSF trypanosomes did not alter either in vitro 235 parasite growth or cell motility in matrix-dependent medium. However, quantitative analyses showed that FLAM8-null trypanosomes were less numerous in extravascular

237 tissues as compared to parental controls. Assuming that parasite motility could be 238 different in tissues and interstitial spaces of extravascular niches with biophysical 239 properties distinct from those in the blood (Bargul et al., 2016; Sun et al., 2018), one 240 cannot exclude that the motility of $\triangle F L A M 8$ knockout parasites might be somehow 241 altered in the extravascular compartment. Intravital imaging for motility analyses at the 242 cell level would be needed in order to confirm this hypothesis.

243 Historically, the majority of studies on T. brucei virulence in experimental infections 244 have considered the blood circulation almost as the sole host compartment parasitized by trypanosomes, whereas extravascular parasite niches and the underlying 
246 exchanges between both compartments have been underestimated for long. The fact

247 that FLAM8-null trypanosomes were not able to disseminate properly over the 248 extravascular compartment could somehow imply defects in the way parasites sense

249 their microenvironment, resulting in an alteration of their extravascular tropism. For 250 example, FLAM8 could be involved in the parasite extravasation process and / or in 251 some subsequent host-parasite interactions promoting their maintenance and 252 development once they have reached the extravascular compartment.

253 To our knowledge, FLAM8 is the first flagellar component affecting parasite 254 dissemination in the extravascular host tissues in vivo. Further investigations on the 255 FLAM8 interactions with other possible partners in the flagellum would help to unravel 256 the roles of this fascinating and essential organelle, especially regarding the 257 modulation of trypanosome distribution in their mammalian hosts, and its potential 258 implications in parasite virulence and transmission. 


\section{Experimental procedures}

\section{Strains, culture and in vitro differentiation}

261 The AnTat 1.1E Paris pleomorphic clone of Trypanosoma brucei brucei was derived

262 from a strain originally isolated from a bushbuck in Uganda in 1966 (Le Ray, Barry, 263 Easton, \& Vickerman, 1977). The monomorphic T. brucei strain Lister 427 (Bohringer 264 \& Hecker, 1974) was also used. All bloodstream forms (BSF) were cultivated in HMI11 medium supplemented with 10\% (v/v) fetal bovine serum (FBS) (Hirumi \& Hirumi, 1989) at $37^{\circ} \mathrm{C}$ in $5 \% \mathrm{CO}_{2}$. For in vitro slender to stumpy BSF differentiation, we used 8-pCPT-2'-O-Me-5'-AMP, a nucleotide analog of 5'-AMP from BIOLOG Life Science Institute (Germany). Briefly, $2 \times 10^{6}$ cultured pleomorphic AnTat 1.1E slender forms were incubated with 8-pCPT-2'-O-Me-5'-AMP $(5 \mu \mathrm{M})$ for $48 \mathrm{~h}$ (Barquilla et al., 2012).

270 For specific experiments, in vitro differentiation of BSF into PCF was performed by 271 transferring freshly-differentiated short stumpy forms into SDM-79 medium 272 supplemented with $10 \%$ (v/v) FBS, $6 \mathrm{mM}$ cis-aconitate and $20 \mathrm{mM}$ glycerol at $27^{\circ} \mathrm{C}$ 273 (Czichos, Nonnengaesser, \& Overath, 1986).

274 Monomorphic BSF "Single Marker" (SM) trypanosomes are derivatives of the Lister 275427 strain, antigenic type MITat 1.2, clone 221a (Doyle, Hirumi, Hirumi, Lupton, \& 276 Cross, 1980), and express the T7 RNA polymerase and tetracycline repressor. 277 FLAM8 ${ }^{R N A i}$ cells express complementary single-stranded RNA corresponding to a 278 fragment of the FLAM8 gene from two tetracycline-inducible T7 promoters facing each 279 other in the pZJM vector (Wang, Morris, Drew, \& Englund, 2000) integrated in the rDNA 280 locus (Wirtz, Leal, Ochatt, \& Cross, 1999). Addition of tetracycline $(1 \mu \mathrm{g} / \mathrm{mL})$ to the 281 medium induces expression of sense and anti-sense RNA strands that can anneal to 282 form double-stranded RNA (dsRNA) and trigger RNAi. For in vivo RNAi studies in mice, 
283 doxycycline hyclate (Sigma Aldrich) was added in sugared drinking water (0.2 g/L 284 doxycycline hyclate combined with $50 \mathrm{~g} / \mathrm{L}$ sucrose).

\section{Generation of FLAM8 RNAi mutants}

286 For the generation of the FLAM8 ${ }^{R N A i}$ cell lines, a 380 bp (nucleotides 6665-7044) 287 fragment of FLAM8 (Tb927.2.5760), flanked by 5' HindIII and 3' Xhol restriction sites 288 to facilitate subsequent cloning, was selected using the RNAit algorithm 289 (http://trypanofan.bioc.cam.ac.uk/software/RNAit.html) in order to ensure that the 290 targeted sequence was distinct from any other genes to avoid any cross-RNAi effects

291 (Redmond, Vadivelu, \& Field, 2003). This FLAM8 DNA fragment was synthesized by

292 GeneCust Europe (Dudelange, Luxembourg) and inserted into the HindIII-Xhol 293 digested pZJM vector (Wang et al., 2000).

294 The pZJM-FLAM8 plasmid was linearized with Notl prior to transfection using 295 nucleofector technology (Lonza, Italy) as described previously (Burkard, Fragoso, \& 296 Roditi, 2007). The cell line was further engineered for endogenous tagging of FLAM8 297 with an mNeonGreen (mNG) at its C-terminal end by using the p3329 plasmid (Kelly 298 et al., 2007), carrying a FLAM8 gene fragment corresponding to FLAM8 ORF 299 nucleotides 8892-9391. Prior to nucleofection, Nrul linearization of p3329-FLAM8300 mNG plasmid was performed.

301 For in vivo experiments in mice, $F L A M 8^{R N A i}$ parasites were finally modified by 302 integrating a plasmid encoding for the chimeric triple reporter which combines the redshifted firefly luciferase PpyREH9 and the tdTomato red fluorescent protein fused with 304 a TY1 tag (Calvo-Alvarez et al., 2018). Transformants were selected with the 305 appropriate antibiotic concentrations: phleomycin $(1 \mu \mathrm{g} / \mathrm{mL})$, blasticidin $(5 \mu \mathrm{g} / \mathrm{mL})$, 306 G418 $(2 \mu \mathrm{g} / \mathrm{mL})$, and puromycin $(0.1 \mu \mathrm{g} / \mathrm{mL})$. Clonal populations were obtained by 
307 limiting dilution. Cell culture growth was monitored with an automatic Muse cell 308 analyser (Merck Millipore, Paris).

\section{Generation of FLAM8 KO mutants}

310 For generating the FLAM8 knockout and rescue cell lines, all insert templates were

311 synthesized by GeneCust Europe (Dudelange, Luxembourg). For breaking the first 312 allele, the 300 first nucleotides of the FLAM8 gene flanking sequences were added on 313 each side of a HYG resistance cassette (Fig. S2). For a complete disruption of the

314 FLAM8 locus, a second selectable marker (PAC) was flanked with the FLAM8-flanking 315 sequence at 5' and by 300 nucleotides of the FLAM8 ORF (nucleotides 501-800) at 3'.

316 For generating an add-back rescue cell line, due to the important size of the FLAM8

317 ORF $(9,228$ nucleotides), the PAC selection marker was replaced by a BLE marker 318 cassette flanked by the 300 first nucleotides of the FLAM85' untranslated region (UTR) 319 and by the nucleotides 1 to 500 of the FLAM8 ORF for reinsertion into the endogenous 320 locus of the knockout cell line. PCR amplifications of the DNA fragments bearing the 321 FLAM8 flanking sequences and the appropriate resistance markers were used for nucleofection and generation of all cell lines. The primers used are listed below: 5'CATGACTTTACGTGTTTGGGCAC-3' (FW, located 82 bp upstream the flanking 5’UTR sequence); 5'-CTTGCTTGTTTCTGTTTCGCAAC-3' (RV, 130 bp downstream the flanking 3'UTR sequence, used to replace one WT allele by HYG resistance 326 cassette); 5'- GCACACTAAAACTCATTGAAAGCC-3' (RV, 926 bp downstream the 327 ATG codon of FLAM8, used for second WT allele replacement by PAC cassette and rescue line generation). All knockout and rescue cell lines were further transfected to express the chimeric triple reporter protein PpyRE9H/TY1/tdTomato for multimodal in

330 vivo imaging approaches as described elsewhere (Calvo-Alvarez et al., 2018).

331 Selection-marker recovery was confirmed by screening individual clones in multi-well 
332 plates. Transformants were selected with the appropriate antibiotic concentrations: phleomycin $(1 \mu \mathrm{g} / \mathrm{mL})$, blasticidin $(5 \mu \mathrm{g} / \mathrm{mL})$, puromycin $(0.1 \mu \mathrm{g} / \mathrm{mL})$ and hygromycin (2.5 $\mu \mathrm{g} / \mathrm{mL})$. Clonal populations were obtained by limiting dilution and cell culture growth was monitored with an automatic Muse cell analyzer (Merck Millipore, Paris). Knockout and rescue cell lines were validated by whole-genome sequencing (BGI, Hong Kong). Briefly, genomic DNA from parental and mutant cell lines were sequenced by the HiSeq4000 sequence system (Illumina), generating about 10 million 100-bp reads and compared to that of the $T$. brucei brucei AnTat 1.1E Paris reference strain. In addition, some validation of the construct integrations in mutants were performed by PCR analysis according to standard protocols (Fig. S2 and Table 1).

\section{Motility analyses}

In silico 2D tracking was performed as previously described (Rotureau, Ooi, Huet, Perrot, \& Bastin, 2014). For each BSF strain, 10 to 20 movies were recorded for 20 seconds (50 ms of exposure). Trypanosomes at $1 \times 10^{6}$ parasites $/ \mathrm{mL}$ were maintained in matrix-dependent $\mathrm{HMI}-11$ medium containing $0,5 \%$ methylcellulose at $37^{\circ} \mathrm{C}$ and were observed under the 10x objective of an inverted DMI-4000B microscope (Leica) coupled to an ORCA-03G (Hamamatsu) or a PRIM95B (Photometrics) camera. Movies were converted with the MPEG Streamclip V.1.9b3 software (Squared 5) and analyzed with the medeaLAB CASA Tracking V.5.5 software (medea AV GmbH). Results were analyzed as mean \pm SD of three independent experiments.

\section{In vitro bioluminescence quantification and analysis}

To perform the parasite density / bioluminescence intensity assay, BSF parasites were counted, centrifuged and resuspended in fresh $\mathrm{HMI}-11$ medium. Then, $100 \mu \mathrm{L}$ of this suspension containing $10^{6}$ parasites were transferred into black clear-bottom 96-well plates and serial 2-fold dilutions were performed in triplicate adjusting the final volume 
to $200 \mu \mathrm{L}$ with $300 \mu \mathrm{g} / \mathrm{mL}$ of beetle luciferin (Promega, France). Luciferase activity was quantified after 10 min of incubation with an IVIS Spectrum imager (PerkinElmer). Imaging data analysis was performed with the Living Image 4.3.1 software (PerkinElmer) by drawing regions of interest with constant size for well plate quantification. Total photon flux was calculated after removal of intensity values from WT parasites and / or parasite-free medium corresponding to the background noise. Results were analyzed as mean \pm SD of three independent experiments.

\section{Mouse infection and ethical statements}

Seven-week-old male BALB/c mice were purchased from Janvier Laboratory (substrain BALB/cAnNRj) and used as models for experimental infection and monitoring of the bioluminescence signal with the IVIS Spectrum imager (PerkinElmer). This study was conducted in strict accordance with the recommendations from the Guide for the Care and Use of Laboratory Animals of the European Union (European Directive 2010/63/UE) and the French Government. The protocol was approved by the "Comité d'éthique en expérimentation animale de l'Institut Pasteur" CETEA 89 (Permit number: 2012-0043 and 2016-0017) and undertaken in compliance with the Institut Pasteur Biosafety Committee (protocol CHSCT 12.131). BR is authorized to perform experiments on vertebrate animals (license \#A-75-2035) and is responsible for all the experiments conducted personally or under his supervision. For in vivo infections, groups of four and three animals (FLAM8 knockdown and knockout-infected mice, respectively) were injected intraperitoneally (IP) with $10^{5}$ slender BSF parasites, washed in TDB (Trypanosome Dilution Buffer: $5 \mathrm{mM} \mathrm{KCl}, 80 \mathrm{mM} \mathrm{NaCl}, 1 \mathrm{mM}$ $\mathrm{MgSO}_{4}{ }^{*} 7 \mathrm{H}_{2} \mathrm{O}, 20 \mathrm{mM} \mathrm{Na}_{2} \mathrm{HPO}_{4}, 2 \mathrm{mM} \mathrm{NaH}_{2} \mathrm{PO}_{4}, 20 \mathrm{mM}$ glucose) and resuspended in $100 \mu$ l of PBS prior animal inoculation. 
In vivo bioluminescence imaging, analysis and parasitemia quantifications

382 Infection with bioluminescent parasites was monitored daily by detecting the

383 bioluminescence signal in whole animals with the IVIS Spectrum imager (PerkinEImer).

384 The equipment consists of a cooled charge-coupled camera mounted on a light-tight

385 chamber with a nose cone delivery device to keep the mice anaesthetized during

386 image acquisition with $1.5-2 \%$ isoflurane. A heated stage is comprised within the IVIS

387 Spectrum imager in order to maintain optimum body temperature. D-luciferin

388 potassium salt (Promega) stock solution was prepared in sterile PBS at $33.33 \mathrm{mg} / \mathrm{mL}$,

389 and stored in a $-20^{\circ} \mathrm{C}$ freezer. To produce bioluminescence, mice were inoculated by

390 the intraperitoneal route with $150 \mu \mathrm{L}$ of D-luciferin stock solution $(250 \mathrm{mg} / \mathrm{Kg}$ body

391 weight). After 10 minutes of incubation to allow substrate dissemination, all mice were

392 anaesthetized in an oxygen-rich induction chamber with $1.5-2 \%$ isoflurane, and images

393 were acquired by using automatic exposure (30 seconds to 5 minutes) depending on

394 signal intensity.

395 Images were analyzed with the Living Image software version 4.3.1 (PerkinElmer).

396 Data were expressed in total photons/second $(\mathrm{p} / \mathrm{s})$ corresponding to the total flux of

397 bioluminescent signal according to the selected area (regions of interest with constant

398 size covering the total body of the mouse). The background noise was removed by

399 subtracting the bioluminescent signal of the control mouse from the infected ones for

400 each acquisition. For parasite dissemination analyses, a minimum value of

401 photons/second $(\mathrm{p} / \mathrm{s})$ was set for all animals in every time point in order to quantify the

402 exact dissemination area (in $\mathrm{cm}^{2}$ ) over the whole animal body. Parasitemia was

403 determined daily following tail bleeds and assayed by automated fluorescent cell

404 counting with a Muse cytometer (Merck-Millipore, detection limit at $10^{2}$ parasites $/ \mathrm{mL}$ )

405 according to the manufacturer's recommendations. The quantification of the total 
406 intravascular parasite population was assessed by calculating the blood volume of all animals according to their body weight and referring to daily parasitemia. To quantify

408 the number of extravascular parasites, a region of interest with a constant size was used to obtain the total number of parasites within the entire animal bodies (total bioluminescence). Subsequently, the total number of parasites present in the vascular system was subtracted, resulting in estimating the total parasite population colonizing

412 the extravascular compartments at a given time point.

\section{Immunofluorescence analysis (IFA)}

414 Cultured parasites were washed twice in TDB and spread directly onto poly-L-lysine 415 coated slides. For methanol fixation, slides were air-dried for 10 min, fixed in methanol at $-20^{\circ} \mathrm{C}$ for $5 \mathrm{~min}$ and rehydrated for $20 \mathrm{~min}$ in PBS. For immunodetection, slides were

417 incubated for $1 \mathrm{~h}$ at $37^{\circ} \mathrm{C}$ with the appropriate dilution of the first antibody in $0.1 \% \mathrm{BSA}$ in PBS. After 3 consecutive 5 min washes in PBS, species and subclass-specific secondary antibodies coupled to the appropriate fluorochrome (Alexa 488, Cy3, Cy5 420 Jackson ImmunoResearch) were diluted $1 / 400$ in PBS containing $0.1 \%$ BSA and were applied for $1 \mathrm{~h}$ at $37^{\circ} \mathrm{C}$. After washing in PBS as indicated above, slides were finally 422 stained with 4',6-diamidino-2-phenylindole (DAPI, $1 \mu \mathrm{g} / \mathrm{mL}$ ) for visualization of 423 kinetoplast and nuclear DNA content, and mounted under cover slips with ProLong 424 antifade reagent (Invitrogen), as previously described (Rotureau et al., 2011). Slides 425 were observed under an epifluorescence DMI4000 microscope (Leica) with a 100x objective (NA 1.4), an EL6000 (Leica) as light excitation source and controlled by the Micro-Manager V1.4.22 software (NIH), and images were acquired using an ORCA03G (Hamamatsu) or a PRIM95B (Photometrics) camera. Images were analyzed with 429 ImageJ V1.8.0 (NIH). The monoclonal antibody mAb25 (anti-mouse IgG2a, 1:10) was 430 used as a flagellum marker as it specifically recognizes the axoneme protein TbSAXO1 
431 (Dacheux et al., 2012). FLAM8 was detected using: i) a specific rabbit serum (1:500)

432 kindly provided by Paul McKean (University of Lancaster, UK), or ii) a monoclonal anti-

433 mNeonGreen antibody (anti-mouse IgG2c, 1:100, ChromoTek). Stumpy BSF were

434 identified at the molecular level with a rabbit polyclonal anti-PAD1 antibody (kindly

435 provided by Keith Matthews, University of Edinburgh; dilution 1:300) (Dean, Marchetti,

436 Kirk, \& Matthews, 2009). In the case of RNAi knockdown experiments, IFA signals

437 were normalized using the signal obtained in non-induced controls as a reference.

438 Measurements, normalization and statistical analyses

439 Standardization of fluorescent signals was carried out by parallel setting of raw

440 integrated density signals in all the images to be compared in ImageJ V1.8.0 (NIH).

441 For clarity purposes, the brightness and contrast of several pictures were adjusted

442 after their analysis in accordance to editorial policies. Statistical analyses and plots

443 were performed with XLSTAT 2019.2.01 (Addinsoft) in Excel 2016 (Microsoft) or Prism

444 V8.2.1 (GraphPad). Statistical analyses include linear regression analyses for

445 bioluminescence / fluorescence intensity vs. parasite density, two-sided ANOVA /

446 ANCOVA tests for growth curves, with Tukey ad-hoc post-tests for inter-group

447 comparison, all at $95 \%$ confidence. 


\section{Acknowledgements}

449 We thank M. Bonhivers, D. Robinson, P. McKean, K. Matthews and K. Gull for

450 providing various plasmids and antibodies. We acknowledge the IP Biolmaging 451 Plateforme for providing access to their equipment. We are grateful to P. Bastin for his 452 strong scientific and human support. We warmly thank P. Bastin, M. Boshart and S. 453 Bonnefoy for their critical reading of the manuscript.

\section{$454 \quad$ Funding}

455 This work was supported by the Institut Pasteur, the French Government 456 Investissement d'Avenir programme - Laboratoire d'Excellence "Integrative Biology of 457 Emerging Infectious Diseases" (ANR-10-LABX-62-IBEID) and the French National 458 Agency for Scientific Research (projects ANR-14-CE14-0019-01 EnTrypa and ANR459 18-CE15-0012 TrypaDerm). None of these funding sources has a direct scientific or 460 editorial role in the present study.

\section{Author contributions}

462 ECA, CT and AC performed the experiments. ECA and BR designed the study, 463 analyzed the data and wrote the manuscript.

\section{Competing interest}

465 All authors declare no financial relationships with any organizations that might have an 466 interest in the submitted work in the previous three years, no other relationships or 467 activities that could appear to have influenced the submitted work, and no other 468 relationships or activities that could appear to have influenced the submitted work. 


\section{References}

Bargul, J. L., Jung, J., McOdimba, F. A., Omogo, C. O., Adung'a, V. O., Kruger, T., . . . Engstler, M. (2016). Species-Specific Adaptations of Trypanosome Morphology and Motility to the Mammalian Host. PLoS Pathog, 12(2), e1005448. doi:10.1371/journal.ppat.1005448

Barquilla, A., Saldivia, M., Diaz, R., Bart, J. M., Vidal, I., Calvo, E., . . Navarro, M. (2012). Third target of rapamycin complex negatively regulates development of quiescence in Trypanosoma brucei. Proc Natl Acad Sci U S A, 109(36), 1439914404. doi:10.1073/pnas.1210465109

Bertiaux, E., Morga, B., Blisnick, T., Rotureau, B., \& Bastin, P. (2018). A Grow-andLock Model for the Control of Flagellum Length in Trypanosomes. Curr Biol. doi:10.1016/j.cub.2018.10.031

Biteau, N., Asencio, C., Izotte, J., Rousseau, B., Fevre, M., Pillay, D., \& Baltz, T. (2016). Trypanosoma brucei gambiense Infections in Mice Lead to Tropism to the Reproductive Organs, and Horizontal and Vertical Transmission. PLoS Negl Trop Dis, 10(1), e0004350. doi:10.1371/journal.pntd.0004350

Bohringer, S., \& Hecker, H. (1974). Quantitative ultrastructural differences between strains of the Tryponasoma brucei subgroup during transformation in blood. $J$ Protozool, 21(5), 694-698. doi:10.1111/j.1550-7408.1974.tb03731.x

Broadhead, R., Dawe, H. R., Farr, H., Griffiths, S., Hart, S. R., Portman, N., . . Gull, K. (2006). Flagellar motility is required for the viability of the bloodstream trypanosome. Nature, 440(7081), 224-227. doi:nature04541 [pii] 10.1038/nature04541 
493 Burkard, G., Fragoso, C. M., \& Roditi, I. (2007). Highly efficient stable transformation

494

495

496

497

498

499

500

501

502

503

504

505

506

507

508

509

510

511

\section{2}

513

514

515

516

517 of bloodstream forms of Trypanosoma brucei. Mol Biochem Parasitol, 153(2), 220-223. doi:10.1016/j.molbiopara.2007.02.008

Caljon, G., Van Den Abbeele, J., Sternberg, J. M., Coosemans, M., De Baetselier, P., \& Magez, S. (2006). Tsetse fly saliva biases the immune response to Th2 and induces anti-vector antibodies that are a useful tool for exposure assessment. Int J Parasitol, 36(9), 1025-1035. doi:10.1016/j.ijpara.2006.05.002

Caljon, G., Van Reet, N., De Trez, C., Vermeersch, M., Perez-Morga, D., \& Van Den Abbeele, J. (2016). The Dermis as a Delivery Site of Trypanosoma brucei for $\begin{array}{llll}\text { Tsetse } \quad \text { Flies. } & \text { PLoS } 1005744 .\end{array}$ doi:10.1371/journal.ppat.1005744

Calvo-Alvarez, E., Cren-Travaillé, C., Crouzols, A., \& Rotureau, B. (2018). A new chimeric triple reporter fusion protein as a tool for in vitro and in vivo multimodal imaging to monitor the development of African trypanosomes and Leishmania parasites. Infect Genet Evol, 63, 391-403. doi:10.1016/j.meegid.2018.01.011

Camara, M., Soumah, A. M., Ilbouldo, H., Travaille, C., Clucas, C., Cooper, A., . . . Rotureau, B. (2020). Extravascular dermal trypanosomes in suspected and confirmed cases of gambiense Human African Trypanosomiasis. Clinical Infectious Diseases. doi:10.1093/cid/ciaa897

Capewell, P., Cren-Travaille, C., Marchesi, F., Johnston, P., Clucas, C., Benson, R. A., .. . MacLeod, A. (2016). The skin is a significant but overlooked anatomical reservoir for vector-borne African trypanosomes. Elife, 5(September2016). doi:10.7554/eLife.17716

Carvalho, T., Trindade, S., Pimenta, S., Santos, A. B., Rijo-Ferreira, F., \& Figueiredo, L. M. (2018). Trypanosoma brucei triggers a marked immune response in male 
reproductive organs. PLoS Negl Trop Dis, 12(8), e0006690. doi:10.1371/journal.pntd.0006690

Czichos, J., Nonnengaesser, C., \& Overath, P. (1986). Trypanosoma brucei: cisaconitate and temperature reduction as triggers of synchronous transformation of bloodstream to procyclic trypomastigotes in vitro. Exp Parasitol, 62(2), 283291. doi:10.1016/0014-4894(86)90033-0

Dacheux, D., Landrein, N., Thonnus, M., Gilbert, G., Sahin, A., Wodrich, H., . . . Bonhivers, M. (2012). A MAP6-related protein is present in protozoa and is involved in flagellum motility. PLoS ONE, 7(2), e31344. doi:10.1371/journal.pone.0031344

Dean, S., Marchetti, R., Kirk, K., \& Matthews, K. R. (2009). A surface transporter family conveys the trypanosome differentiation signal. Nature, 459(7244), 213-217. doi:nature07997 [pii] 10.1038/nature07997

Doyle, J. J., Hirumi, H., Hirumi, K., Lupton, E. N., \& Cross, G. A. (1980). Antigenic variation in clones of animal-infective Trypanosoma brucei derived and maintained in vitro. Parasitology, $\quad 80(2), \quad 359-369$. doi:10.1017/s0031182000000810

Fort, C., Bonnefoy, S., Kohl, L., \& Bastin, P. (2016). Intraflagellar transport is required for the maintenance of the trypanosome flagellum composition but not its length. J Cell Sci, 129(15), 3026-3041 . doi:10.1242/jcs.188227

Goodwin, L. G. (1970). The pathology of African trypanosomiasis. Trans $R$ Soc Trop Med Hyg, 64(6), 797-817.

Hirumi, H., \& Hirumi, K. (1989). Continuous cultivation of Trypanosoma brucei blood stream forms in a medium containing a low concentration of serum protein without feeder cell layers. J Parasitol, 75(6), 985-989. 
543 Kelly, S., Reed, J., Kramer, S., Ellis, L., Webb, H., Sunter, J., . . Carrington, M. (2007). Functional genomics in Trypanosoma brucei: a collection of vectors for the expression of tagged proteins from endogenous and ectopic gene loci. Mol Biochem Parasitol, 154(1), 103-109. doi:S0166-6851(07)00099-0 [pii] 10.1016/j.molbiopara.2007.03.012

Kohl, L., Robinson, D., \& Bastin, P. (2003). Novel roles for the flagellum in cell morphogenesis and cytokinesis of trypanosomes. EMBO J, 22(20), 5336-5346. doi:10.1093/emboj/cdg518

Langousis, G., \& Hill, K. L. (2014). Motility and more: the flagellum of Trypanosoma brucei. Nat Rev Microbiol, 12(7), 505-518. doi:10.1038/nrmicro3274

Le Ray, D., Barry, J. D., Easton, C., \& Vickerman, K. (1977). First tsetse fly transmission of the "AnTat" serodeme of Trypanosoma brucei. Ann Soc Belg Med Trop, 57(4-5), 369-381.

MacGregor, P., Szoor, B., Savill, N. J., \& Matthews, K. R. (2012). Trypanosomal immune evasion, chronicity and transmission: an elegant balancing act. Nat Rev Microbiol, 10(6), 431-438. doi:10.1038/nrmicro2779

Ooi, C. P., \& Bastin, P. (2013). More than meets the eye: understanding Trypanosoma brucei morphology in the tsetse. Front Cell Infect Microbiol, 3, 71. doi:10.3389/fcimb.2013.00071

Redmond, S., Vadivelu, J., \& Field, M. C. (2003). RNAit: an automated web-based tool for the selection of RNAi targets in Trypanosoma brucei. Mol Biochem Parasitol, 128(1), 115-118. doi:10.1016/s0166-6851(03)00045-8

Roditi, I., Schumann, G., \& Naguleswaran, A. (2016). Environmental sensing by African trypanosomes. Curr Opin Microbiol, 32, 26-30. doi:10.1016/j.mib.2016.04.011 
Rojas, F., Silvester, E., Young, J., Milne, R., Tettey, M., Houston, D. R., . . Matthews, K. R. (2018). Oligopeptide Signaling through TbGPR89 Drives Trypanosome Quorum Sensing. Cell. doi:10.1016/j.cell.2018.10.041

571

Rotureau, B., Morales, M. A., Bastin, P., \& Spath, G. F. (2009). The flagellum-MAP kinase connection in Trypanosomatids: a key sensory role in parasite signaling and development? Cell Microbiol, 11(5), 710-718. doi:CMl1295 [pii] 10.1111/j.1462-5822.2009.01295.x

Rotureau, B., Ooi, C. P., Huet, D., Perrot, S., \& Bastin, P. (2014). Forward motility is essential for trypanosome infection in the tsetse fly. Cell Microbiol, 16(3), 425433. doi:10.1111/cmi.12230

Rotureau, B., Subota, I., \& Bastin, P. (2011). Molecular bases of cytoskeleton plasticity during the Trypanosoma brucei parasite cycle. Cell Microbiol, 13(5), 705-716. doi:10.1111/j.1462-5822.2010.01566.x

Shimogawa, M. M., Ray, S. S., Kisalu, N., Zhang, Y., Geng, Q., Ozcan, A., \& Hill, K. L. (2018). Parasite motility is critical for virulence of African trypanosomes. Sci Rep, 8(1), 9122. doi:10.1038/s41598-018-27228-0

Smith, T. K., Bringaud, F., Nolan, D. P., \& Figueiredo, L. M. (2017). Metabolic reprogramming during the Trypanosoma brucei life cycle. F1000Res, 6. doi:10.12688/f1000research.10342.2

Subota, I., Julkowska, D., Vincensini, L., Reeg, N., Buisson, J., Blisnick, T., . . Bastin, P. (2014). Proteomic analysis of intact flagella of procyclic Trypanosoma brucei cells identifies novel flagellar proteins with unique sub-localisation and dynamics. Mol Cell Proteomics. doi:10.1074/mcp.M113.033357 
Sun, S. Y., Kaelber, J. T., Chen, M., Dong, X., Nematbakhsh, Y., Shi, J., . . He, C. Y. (2018). Flagellum couples cell shape to motility in Trypanosoma brucei. Proc Natl Acad Sci U S A, 115(26), E5916-E5925. doi:10.1073/pnas.1722618115

594 Tetley, L., \& Vickerman, K. (1985). Differentiation in Trypanosoma brucei: hostparasite cell junctions and their persistence during acquisition of the variable antigen coat. J Cell Sci, 74, 1-19.

Trindade, S., Rijo-Ferreira, F., Carvalho, T., Pinto-Neves, D., Guegan, F., ArestaBranco, F., ... Figueiredo, L. M. (2016). Trypanosoma brucei Parasites Occupy and Functionally Adapt to the Adipose Tissue in Mice. Cell Host Microbe. doi:10.1016/j.chom.2016.05.002

Wang, Z., Morris, J. C., Drew, M. E., \& Englund, P. T. (2000). Inhibition of 602 Trypanosoma brucei gene expression by RNA interference using an integratable vector with opposing T7 promoters. J Biol Chem, 275(51), 4017440179. doi:10.1074/jbc.M008405200

Wirtz, E., Leal, S., Ochatt, C., \& Cross, G. A. (1999). A tightly regulated inducible 606 expression system for conditional gene knock-outs and dominant-negative genetics in Trypanosoma brucei. Mol Biochem Parasitol, 99(1), 89-101.

608 


\section{Figure legends}

610

611 Figure 1. Characterization of the FLAM8 ${ }^{R N A i}$ strains in vitro. A) Expression of

612 FLAM8 mRNA assessed by RT-PCR by the comparative $\Delta \Delta \mathrm{C}_{\mathrm{T}}$ method in control, non-

613 induced and induced FLAM8 ${ }^{R N A i}$ parasites (72 h). B) Immunofluorescence pictures of

614 non-induced (upper panels) and induced (bottom panels) FLAM8 ${ }^{\text {RNAi }}$ FLAM8::mNG

615 BSF during $72 \mathrm{~h}$. Methanol-fixed trypanosomes were stained with an anti-mNG

616 antibody (green) and DAPI for DNA content (blue). The scale bars represent $5 \mu \mathrm{m}$. C)

617 Growth curves of control, non-induced and induced FLAM8 ${ }^{R N A i}$ BSF parasites. All cell

618 lines received $1 \mu \mathrm{g}$ tetracycline during 6 days. Control parasites are Lister 427 "Single

619 Marker" BSF parasites that do not bear the pZJM-FLAM8 plasmid for RNAi silencing.

620 Results represent the mean ( \pm standard deviation, SD) of three independent 621 experiments. 
622 Figure 2. Functional investigations on $F L A M 8^{R N A i}$ parasites in vivo in the

623 mammalian host. Groups of 4 BALB/c mice were injected intraperitoneally with either

624 control, non-induced or induced FLAM8 ${ }^{R N A i}$ BSF trypanosomes. One PBS-injected

$625 \mathrm{BALB} / \mathrm{C}$ animal was used as negative control. A) Number of parasites in the blood

626 (intravascular, IV) of infected BALB/c mice during the course of the infection (5 days).

627 B) Number of parasites in the extravascular compartment (extravascular, EV) of the

628 same mice. C, D, E) Comparison of the number of trypanosomes present in the blood

629 circulation (IV, continuous line) and in extravascular tissues (EV, dotted line) during

630 the entire experimental infection. Significant differences are indicated with * $(p<0.05)$.

631 F) Dissemination of control, non-induced and induced FLAM8 ${ }^{R N A i}$ parasites, measured

632 over the entire animal body (in $\mathrm{cm}^{2}$ ) through the total bioluminescent surface, during

633 the entire infection course. Results represent means \pm standard deviation (SD). G)

634 Representative normalized in vivo images of the bioluminescence radiance signal (in

635 photons / second / $\mathrm{cm}^{2}$ / steradian) emitted from BALB/c mice infected with control,

636 non-induced and induced $F L A M 8^{R N A i}$ parasites 4 days post-infection (non-infected

637 technical control mice were negative for bioluminescence, not shown). 
638 Figure 3. Characterization of the $\triangle F L A M 8$ null mutants in vitro. A)

639 Immunofluorescence pictures of parental, $\triangle F L A M 8$ knockout and rescue pleomorphic

640 BSF parasites labelled with the anti-FLAM8 (green) and mAb25 (axoneme in magenta)

641 antibodies, DAPI staining for DNA content (blue). Scale bars show $5 \mu \mathrm{m}$. B) Growth

642 curve of parental, $\triangle F L A M 8$ and rescue pleomorphic BSF trypanosome cell lines over

6436 consecutive days. C) Measurements of the flagellum length based on the axonemal

644 marker mAb25 profiles in parental, $\triangle F L A M 8$ and rescue parasites. No statistical

645 differences were found. D, E) Motility tracking analysis showing the average speeds

646 (D) and linearity (E) of BSF cell lines in matrix-dependent culture medium. No statistical

647 differences were observed. The number of parasites considered for quantifications (N)

648 is indicated below graphs (C), (D) and (E). Results represent the mean \pm standard 649 deviation (SD) of three independent experiments. 
652 one parental, three $\triangle F L A M 8$ null subclones or one rescue strains. One PBS-injected

653 BALB/c animal was used as negative control. A) Normalized in vivo images of the 654 bioluminescence radiance intensity (in photons / second / $\mathrm{cm}^{2} /$ steradian) emitted 8 days post-infection in BALB/c mice infected with parental, $\triangle F L A M 8$ or rescue parasites

656 (non-infected control mice C- were negative for bioluminescence). B) Representative 657 immunofluorescence pictures of transmissible short stumpy parasites found in the 658 blood of BALB/c mice infected with parental, $\triangle F L A M 8$ and rescue parasites after the 659 first peak of parasitemia. Anti-PAD1 antibody was used as a surface-exposed 660 differentiation marker (red), DAPI staining for DNA content (blue). Scale bars represent $6615 \mu \mathrm{m}$. C) Total number of parasites in the blood of infected mice (intravascular, IV) 662 during the course of the infection (4 weeks). Statistically significant differences 663 between the parental strain and $\triangle F L A M 8$ subclones are indicated with * $(p<0.0005)$. D) 664 Total number of extravascular (EV) trypanosomes in the same mice. Statistically 665 significant differences between the parental strain and $\triangle F L A M 8$ subclones are 666 indicated with ${ }^{*}(\mathrm{p}<0.0005)$. E) Dissemination of the parental, $\triangle F L A M 8$ subclones and 667 rescue parasite strains, measured over the entire animal body (in $\mathrm{cm}^{2}$ ) through the 668 total surface of bioluminescent signal, during the entire infection course. Statistically 669 significant differences between the parental strain and $\triangle F L A M 8$ subclones are 670 indicated with ${ }^{*}(p<0.0005)$. Results represent means \pm standard deviation (SD). F) 671 Ratio between the total number of extravascular parasites quantified in individual 672 dissected organs and the corresponding values of parasitemia at day 27 post infection 673 in BALB/c mice infected with parental, $\triangle F L A M 8$ null subclones or rescue pleomorphic 674 BSF parasites. Only one statistically significant difference indicated with ${ }^{*}(p<0.0005)$ 
bioRxiv preprint doi: https://doi.org/10.1101/2021.01.08.425862; this version posted March 9, 2021. The copyright holder for this preprint (which was not certified by peer review) is the author/funder. All rights reserved. No reuse allowed without permission.

675 was observed when comparing the skin of mice infected with $\triangle F L A M 8$ and parental

676 strains. 


\section{Figure 1}

A
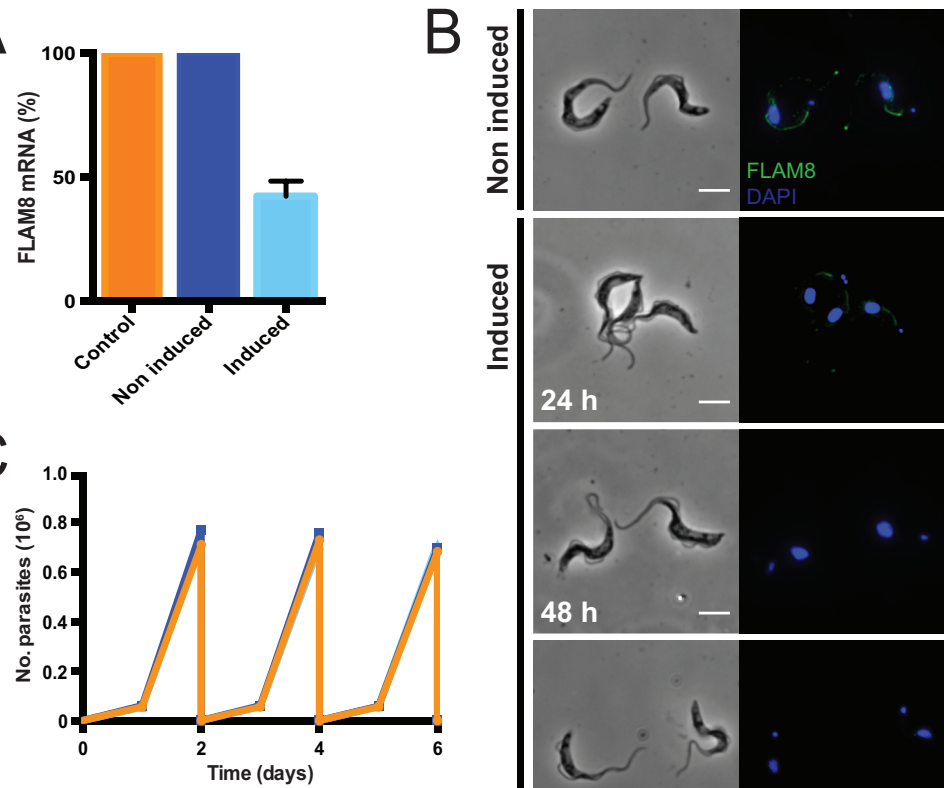

$\leftrightarrow$ Control Non induced $\rightarrow$ Induced

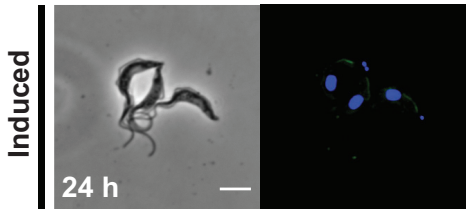

$48 h$

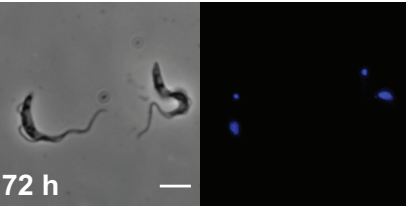


$A$

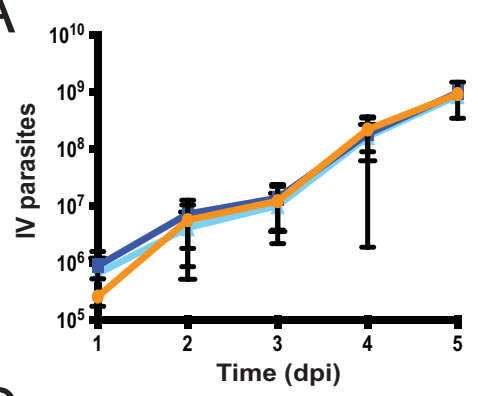

6
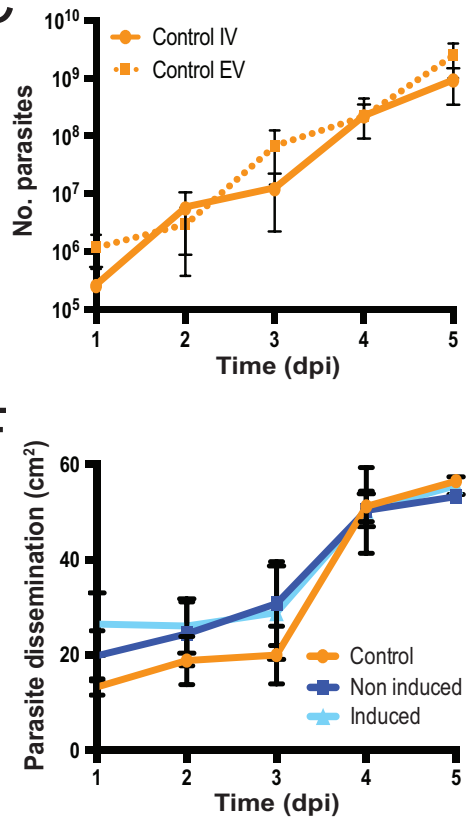

B

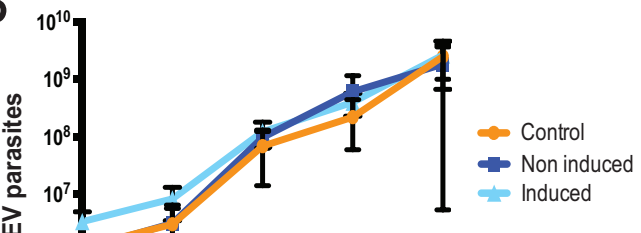

$D$

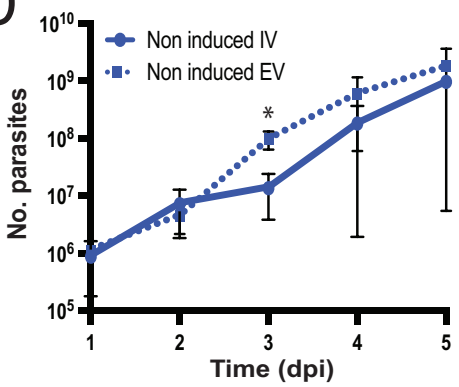

E

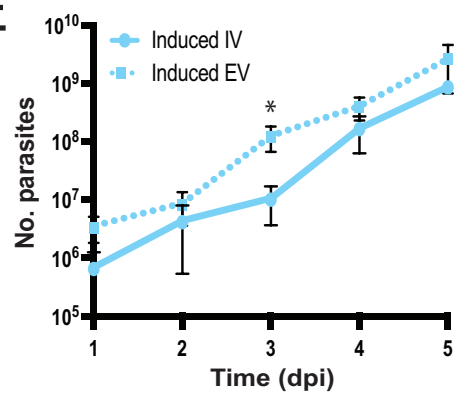

G
Non induced

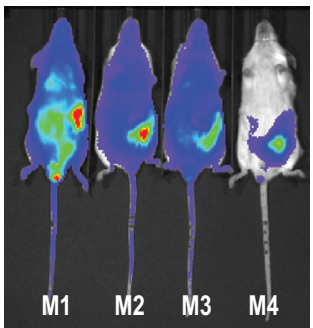

Induced
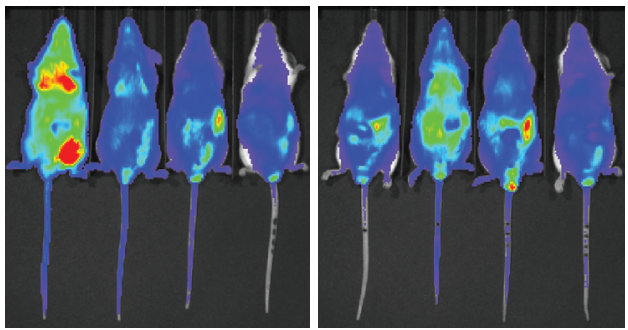

Radiance (p/sec/cm²/sr, $10^{8}$ ) 
Figure 3

A Parental $\triangle F L A M 8$ Rescue B

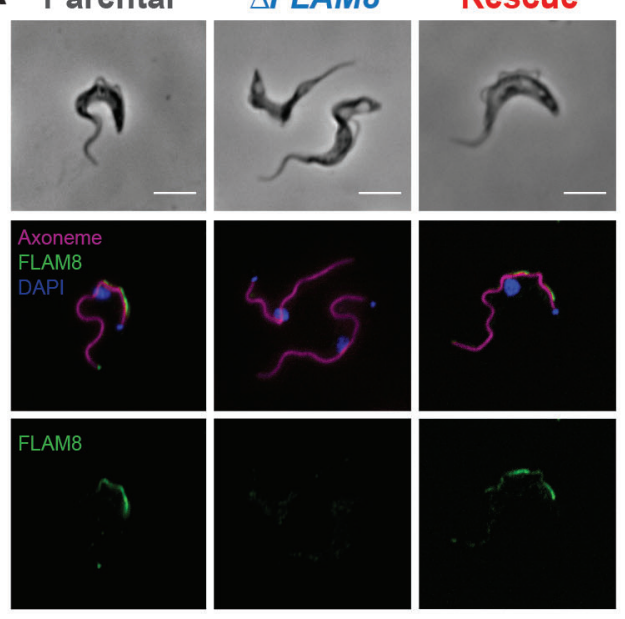

B

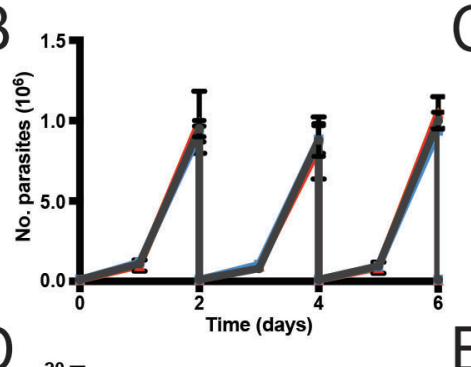

C

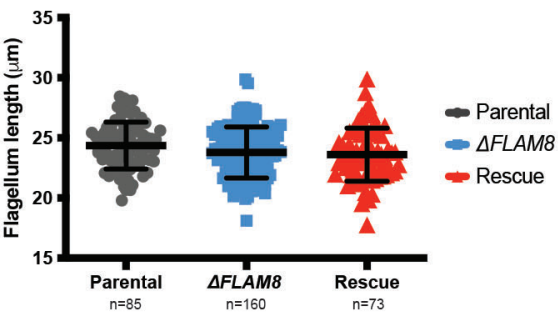

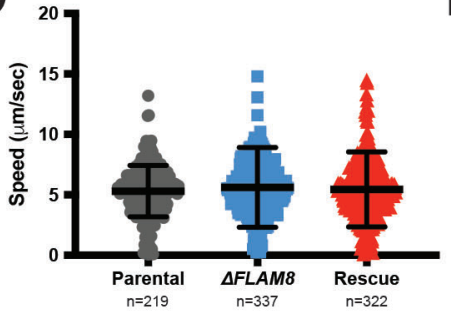

E

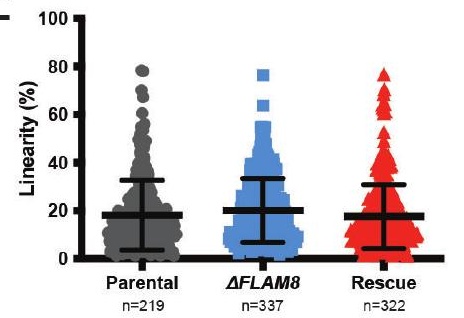


Figure 4

A

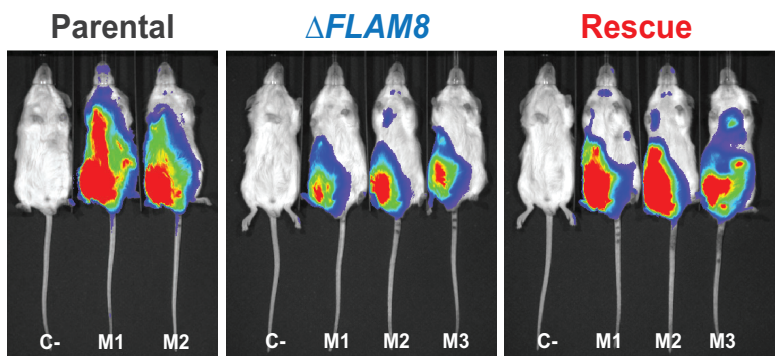

B
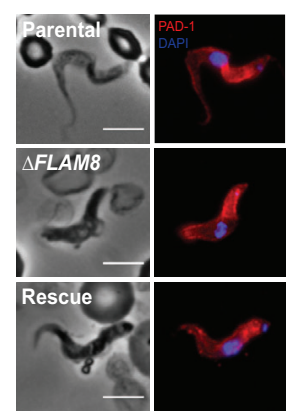

Radiance (p/sec/cm $\left.2 / \mathrm{sr}, 10^{9}\right)$

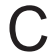

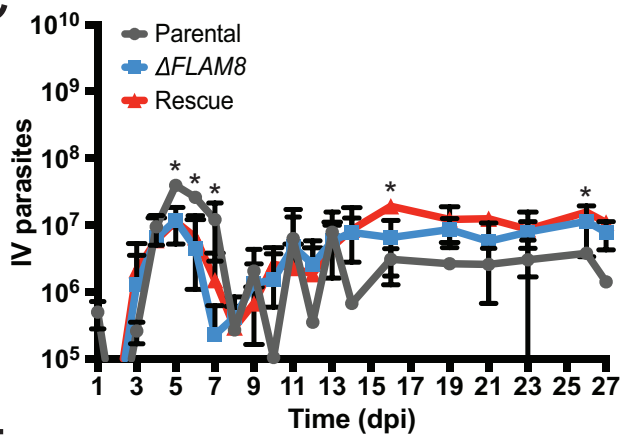



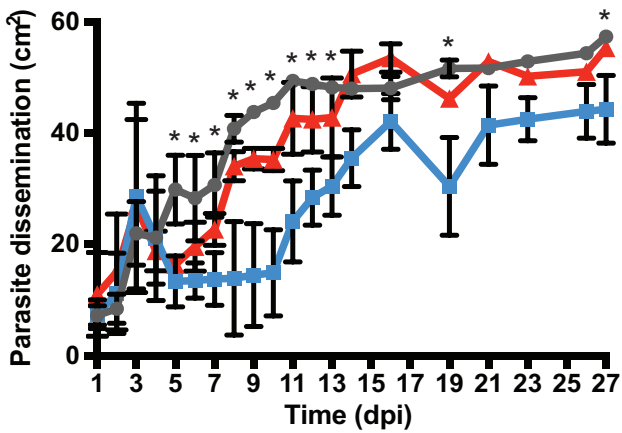

D

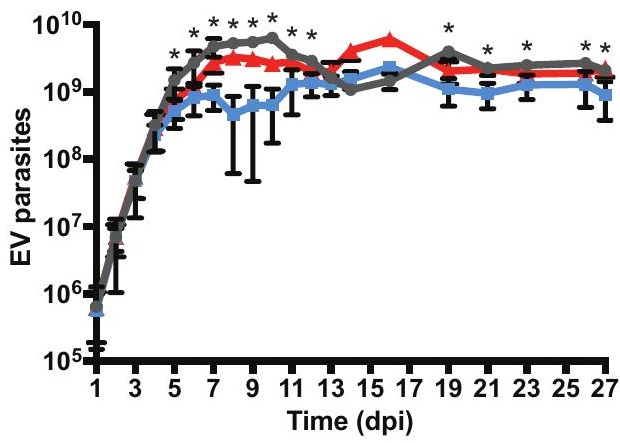

27 dpi

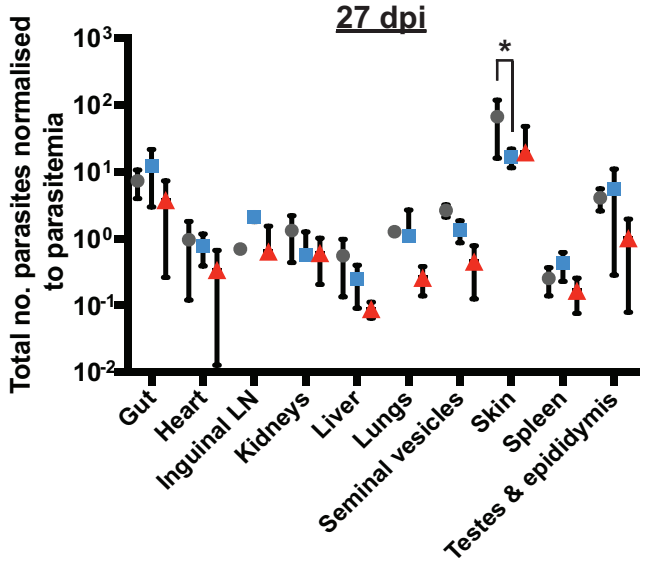


Table 1. Oligonucleotides used for PCR validation of the $\triangle F L A M 8$ knockout and rescue cell lines.

\begin{tabular}{|c|c|c|c|}
\hline Primer & Sequence & Target & \\
\hline 1 & CATGACTTTACGTGTTTGGGCAC & $\begin{array}{l}\text { FLAM8 WT allele (82 nt } \\
\text { upstream 5'UTR) }\end{array}$ & $\mathrm{F}$ \\
\hline 2 & GCACACTAAAACTCATTGAAAGCC & $\begin{array}{l}\text { FLAM8 WT allele (926 nt } \\
\text { downstream ATG) }\end{array}$ & $\mathrm{R}$ \\
\hline 3 & CGTCCGAGGGCAAAGGAATAG & Hygromycin cassette & $\mathrm{R}$ \\
\hline 4 & GACCGCGCACCTGGTGCATG & Puromycin cassette & $\mathrm{R}$ \\
\hline 5 & GTGGCCGAGGAGCAGGACTGA & Phleomycin cassette & $\mathrm{R}$ \\
\hline
\end{tabular}

Orientation of primers: $F$, forward; $R$, reverse. 